\title{
A preliminary study on the use of haptic feedback to assist users with impaired arm coordination during mouse interactions
}

\author{
N.G. Tsagarakis* and D.G. Caldwell \\ Italian Institute of Technology (IIT), Genova, Italy
}

\begin{abstract}
Physical movement impairments caused by central nervous system dysfunction or by muscle spasms generated from other neurological damage or dysfunction can often make it difficult or impossible for affected individuals to interact with computer generated environments using the conventional mouse interfaces. This work investigates the use of a 2 dimensional haptic device as an assistive robotic aid to minimize the effects of the pathological absence of motor control on the upper limb in impaired users while using a mouse interface. The haptic system used in this research is a two degree of freedom (DOF) Pantograph planar device. To detect the intended user motion, the device is equipped with force sensing allowing the monitoring of the user applied loads. Impedance based techniques are used to develop a "clumsy" motion suppression control system. The erratic motion suppression techniques and the experimental system setup are evaluated in two dimensional tracking tasks using a human subject with failure of the gross coordination of the upper limb muscle movements resulting from a disorder called 'Muscle Ataxia'. The results presented demonstrate the ability of the system to improve the tracking performance of the impaired user while interacting with a simple computer generated 2D space.
\end{abstract}

Keywords: Assistive, haptic, computer, interface

\section{Introduction}

In recent years, there has been a considerable body of work directed towards the development of rehabilitation or power and motion coordination assisted robotic devices, and as a result, a wide and diverse range of systems has been produced. These systems range from simple passive link orthoses to fully powered exoskeletons. They use a variety of actuation methods and control strategies and they are targeted at assisting with the recovery from different injuries or to help compen-

*Corresponding author: N.G. Tsagarakis, Italian Institute of Technology (IIT), Genova, 16163, Italy. E-mail: nikos.tsagarakis@ iit.it. sate for various disabilities or pathological conditions such as; Parkinson's disease, muscular dystrophy, muscle ataxia, spinal cord injuries, stroke and cerebral palsy. Each of these disabilities are typically associated with symptoms such as reduced strength, restricted or irregular jerky movements, poor motion coordination and a continuum of impairments involving spasms and tremors. In many situations these physical impairments can make it difficult or impossible for the individuals to interact with computer generated environments using the conventional mouse interfaces $[1,2]$ thus restricting the capacity of suffers take advantage of developments in technology or work. There is a strong belief that assistive robotic devices with the capability to suppress unsteady motions, while at the same time 
providing assistance for weak movements, may help this population to complete motions/tasks that cannot be executed due to the impaired coordinated or through insufficient muscle strength. Based on this hypothesis extensive research efforts, in the last 10-15 years, have focused on the evaluation of robotic systems as aid systems for improving the independence or for individual rehabilitation.

In particular, the efficacy of human machine interfaces such as force feedback mice [3, 4] has been evaluated in GUI interaction tasks. Velocity dependent force feedback has been evaluated in a number of other projects to damp erratic motions [5-7]. It has been shown that increasing the viscous damping helps to reduce the level of sudden motions (spasms) but at the same time increased resistance to voluntary movement may occur. More recently, other mechatronic aids [8-13] have been used in all areas of physical therapy, rehabilitation, motion coordination assistance and performance evaluation.

This work introduces a system that aims to improve the capability and efficiency of people with motion impairments and particularly those with the pathological disorder called muscle 'Ataxia' (a relatively rare disorder with about 20,000 cases in Europe and North America and 3000-5000 in US, 1/50,000) while interacting with GUIs. Ataxia (from Greek ataxia, meaning failure to put in order) is a neurological symptom associated with unsteady and awkward motion of the limbs or trunk due to a failure of the gross coordination of muscle movements. Ataxia often occurs when parts of the nervous system that control movement are damaged. People with ataxia experience a failure of muscle control in their arms and legs, resulting in a lack of balance and coordination or a disturbance of gait. While the term ataxia is primarily used to describe this set of symptoms, it is sometimes also used to refer to a family of disorders. It is not, however, a specific diagnosis [14]. The above symptoms arising from muscle ataxia can make simple keyboard and mouse use for computer interaction difficult or even impossible (Table 1).

Table 1

Specifications of the Planar Haptic device

\begin{tabular}{lc}
\hline Property & Value \\
\hline Actuator & $40 \mathrm{~W}$ DC motors \\
Position resolution & $<0.05 \mathrm{~mm}$ \\
Peak force & $26 \mathrm{~N}$ \\
Continues stall force & $3.9 \mathrm{~N}$ \\
Workspace & $340 \times 250 \mathrm{~mm}$ \\
\hline
\end{tabular}

A haptic assistive device and its control are proposed for improving gross and fine cursor based motions of impaired individuals when interacting with a computer generated environment. This is achieved by a combined assistive/suppressive motion control scheme based on impedance techniques. The robotic device used is a two degrees of freedom (2-DOF) pantograph planar mechanism. The end-tip of the robotic system has been attached to a traditional mouse device to form the complete "Mouse Motion Assistive" interface. The device is instrumented with a force sensor which is used to provide information about the subject's intended motion by monitoring the interaction forces between the device and the user's hand. Evaluation of the device was accomplished on a subject with failure of the gross coordination of the upper limb. The experiments demonstrated that the system can be used efficiently to filter the erratic motions of the impaired subject while interacting with a PC unit in 2D space in a manner similar to mouse interactions.

The paper is organized as follows: Section II presents the assistive haptic device. Section III introduces the principle of the assistive device control scheme, while the experimental system setup is described in section IV. Finally, the results from the trials with the impaired subject are depicted in Section V while Section VI addresses the conclusions and comments on further developments.

\section{Description of the assistive haptic system}

\subsection{Mechanical configuration}

To assist users suffering movement coordination difficulties that limit their capacity to interact with a computer, particularly using interfaces such as a mouse, a customised force feedback device was used $[15,16]$. This is a device operating in a two dimensional space which is sufficient as GUI based interaction using a mouse only involves two dimensional movements. A picture of the custom device formed as a five bar pantograph system is shown in Fig. 1.

The dimensions of the system (links) were specified to permit the end-tip of the device to cover the necessary range of motion usually seen in mouse based interactions. The device is powered by two $40 \mathrm{~W}$ DC-motors from Portescap (Model 25GT2R82-222E) through a capstan cable transmission which provides a 26:1 gear ratio with minimum friction and excellent back drivability. Incremental magnetic encoders 


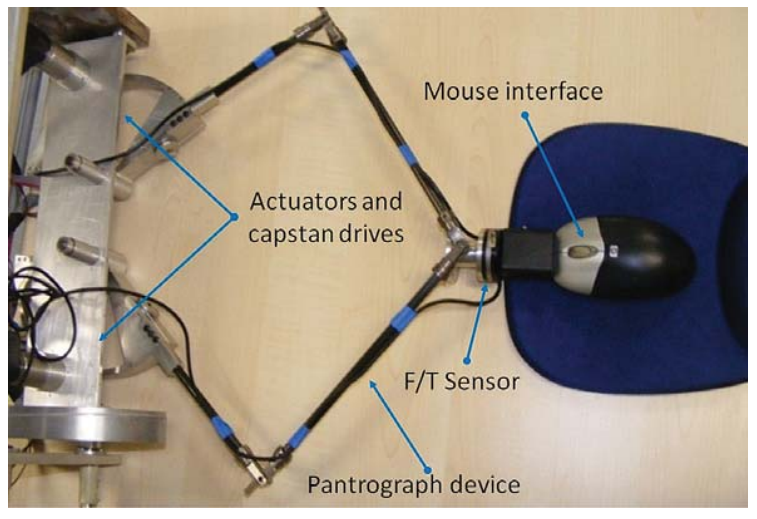

Fig. 1. Picture of the planar pantograph haptic system and its integration with a mouse device.

placed on the motors side monitor the position changes of the device tip while custom device semiconductor strain gauge based torque sensors at the output shaft of each actuator permit the measurement of the actuator's torque. The prototype device has the following specifications:

The prototype haptic system is a relative inexpensive haptic system with a cost of $1300 €$ which includes the fabrication of mechanical parts, actuators and power drives. The haptic system end-tip has been interfaced with a mouse device to form the assistive interface for the GUI based interactions. To allow the monitoring of the exchange of forces between the assistive system and user's hand a force sensor (ATI Mini40) has been installed between the mouse and the haptic device, Fig. 1. The additional cost of the force torque sensor (about $5000 €$ ) can be significantly reduced by replacing it with a two axis load cell with a price of about $400 €$ (although a 2 DOF load cell is sufficient, the 6DOF sensor was utilized in this study as it was an already available device).

\subsection{Device kinematics and dynamics}

The kinematic configuration of the assistive haptic interface is presented in Fig. 2. The position vector of the interface tip is denoted as ${ }^{0} \mathbf{x}$. This distal joint position can be represented as a function of the active joint angles $\mathbf{q}_{\mathbf{i}}, \mathbf{i}=1, \ldots, 2$ and the upper link angle $\mathrm{q}_{\mathrm{u}}$ and is given by

$$
{ }^{0} \mathbf{x}=\left[\begin{array}{c}
\mathrm{a}+\mathrm{L}_{1} \mathrm{c}_{1}+\mathrm{L}_{2} \mathrm{c}_{u} \\
\left(\mathrm{~L}_{1} \mathrm{~s}_{1}+\mathrm{L}_{2} \mathrm{~S}_{u}\right)
\end{array}\right]
$$

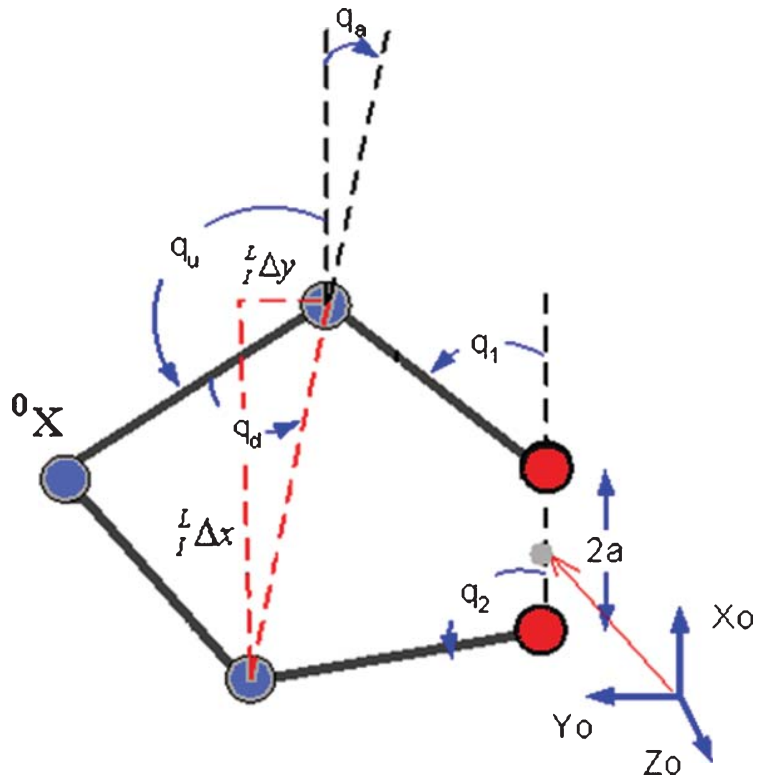

Fig. 2. Device kinematic geometry.

where the upper link angle $\mathbf{q}_{\mathrm{u}}$ can be determined using five bar mechanism geometry. From Fig. $2 \mathrm{q}_{\mathrm{u}}=$ $\pi-q_{d}-q_{a}$ where $q_{d}$ is the angle between the outer link and the line that joins the distal joints of the inner links, and $\mathrm{q}_{\mathrm{a}}$ is the angle between the horizontal line and the line joining the distal joints of the inner links of the five bar mechanism. The angle $\mathrm{q}_{d}$ is computed from the triangle formed by the outer links and the line joining the distal joints of the inner links therefore $\mathrm{q}_{\mathrm{d}}=\mathrm{a} \cos \left(\mathrm{d} / 2 \mathrm{~L}_{2}\right)$ while the angle $\mathrm{q}_{\mathrm{a}}$ is given by $\mathrm{q}_{\mathrm{a}}=\mathrm{a} \tan (\Delta \mathrm{y} / \Delta \mathrm{x})$ where $\mathbf{L}_{2}$ is the length of the distal link and $\Delta \mathrm{x}$ and $\Delta \mathrm{y}$ are the $\mathrm{x}, \mathrm{y}$ distances between the distal joints of the inner links. This finally gives $\mathrm{q}_{\mathrm{u}}=\pi-\mathrm{a} \cos \left(\mathrm{d} / 2 \mathrm{~L}_{2}\right)-\mathrm{a} \tan (\Delta \mathrm{y} / \Delta \mathrm{x})$.

Substituting $\mathrm{q}_{\mathrm{u}}$ in (1) and directly differentiating the final expression of the forward kinematics yields the device Jacobian which relates the handle tip velocity as a function of the two active joint velocities.

$$
{ }^{\mathbf{o}} \mathbf{v}=\mathbf{J} \dot{\mathbf{q}}
$$

The overall dynamic behaviour of the haptic device is described by the following formula.

$$
\mathbf{M}(\mathbf{q}) \ddot{\mathbf{q}}+\mathbf{V}(\mathbf{q}, \dot{\mathbf{q}})+\mathbf{B}(\dot{\mathbf{q}})+\mathbf{J}^{\mathbf{T}} \mathbf{F}_{\mathbf{d}}=\boldsymbol{\tau}
$$

where the newly introduced notations describe; $\boldsymbol{\tau}$ the joint torque vector, $\mathbf{M}(\mathbf{q})$ the inertia matrix, $\mathbf{V}(\mathbf{q}, \dot{\mathbf{q}})$ the coriolis/centripetal vector, $\mathbf{F}(\dot{\mathbf{q}})$ the friction vector, $\mathbf{F}_{\mathbf{d}}$ 

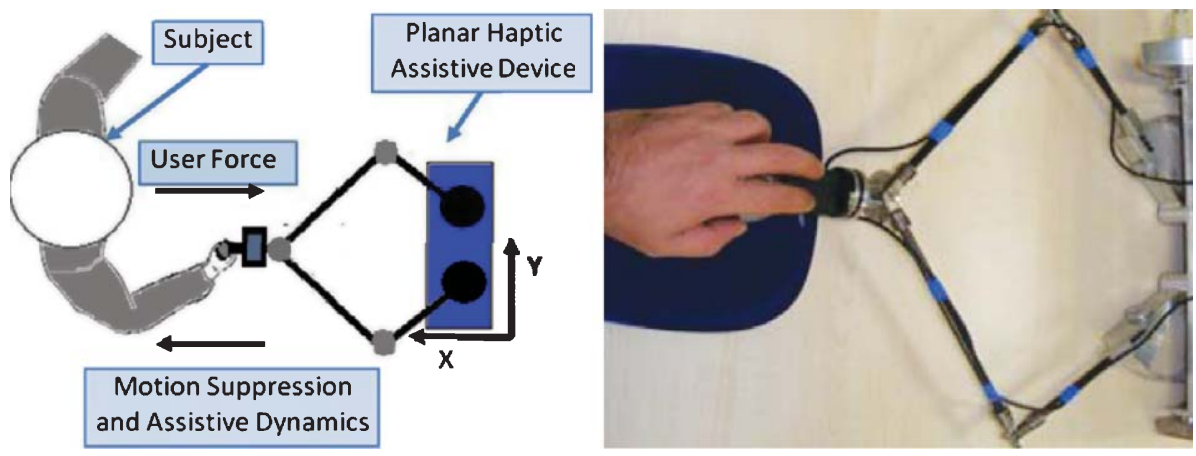

Fig. 3. Schematic diagram of the subject and the planar haptic device.

is the force that the device generates at the end-tip and $\mathbf{J}^{\mathbf{T}}$ is the transpose Jacobian of the haptic device.

\section{The assistive controller for the haptic device}

For this application the haptic device and particularly its control scheme should assist a subject with motion coordination difficulties to interact with GUIs. More particularly, in the case of a person with weakened muscles, the control scheme should be able to assist the motion. In addition, for a person with tremor or unsteady motion intervals the device should also be capable of damping these clumsy motions. We employed an impedance control scheme to achieve these two functionalities. Equation (3) can be used to describe the interaction between the subject and the haptic device. Considering the scenario described in Fig. 3, where the subject's hand is attached to the device tip via a force sensor, let $\mathbf{F}_{\mathbf{s}}$ denotes the force that the subject exerts on the device tip, while $\mathbf{F}_{\mathbf{d}}$ is the force that the device applies to the subject and $\mathbf{Z}_{\mathbf{E}}(\mathbf{s})$ is the systems desired mechanical impedance. To make the pair (Haptic Device + subject's hand) motion follow the desired assistive/suppression impedance dynamics the expression (4) below must be applied.

$$
\begin{aligned}
\mathbf{Z}_{\mathbf{E}}(\mathbf{s})\left(\mathbf{x}-\mathbf{x}_{\mathbf{E}}\right)= & \mathbf{M}_{\mathbf{E}} \ddot{\mathbf{x}}+\mathbf{B}_{\mathbf{E}} \dot{\mathbf{x}} \\
& +\mathbf{K}_{\mathbf{E}}\left(\mathbf{x}-\mathbf{x}_{\mathbf{E}}\right)=\mathbf{F}_{\mathbf{s}}
\end{aligned}
$$

where $\mathbf{M}_{\mathbf{E}}, \mathbf{B}_{\mathbf{E}}, \mathbf{K}_{\mathbf{E}}$ represent the inertia, damping and stiffness matrix coefficients while $\mathbf{x}_{\mathbf{E}}$ is the impedance equilibrium position. The above equation defines the desired characteristics of the motion of the pair (Haptic Device + subject's hand).

Having specified the desired behaviour of the system the control law now can be derived by eliminating $\ddot{\mathbf{x}}$ and $\ddot{\mathbf{q}}$ from (3) and (4). To do this the following equations, which relate the velocities and accelerations of the assistive haptic device end-point to the velocities and accelerations in joint space are introduced.

$$
\begin{aligned}
& \dot{\mathbf{x}}=\mathbf{J} \dot{\mathbf{q}} \\
& \ddot{\mathbf{x}}=\mathbf{J} \ddot{\mathbf{q}}+\dot{\mathbf{J}} \dot{\mathbf{q}}
\end{aligned}
$$

Solving (4) and (6) for $\ddot{\mathbf{x}}$ and $\ddot{\mathbf{q}}$ respectively gives:

$$
\begin{aligned}
& \ddot{\mathbf{x}}=\mathbf{M}_{\mathbf{E}}^{-1}\left(\mathbf{F}_{\mathbf{H}}-\mathbf{B}_{\mathbf{E}} \dot{\mathbf{x}}-\mathbf{K}_{\mathbf{E}}\left(\mathbf{x}-\mathbf{x}_{\mathbf{E}}\right)\right) \\
& \ddot{\mathbf{q}}=\mathbf{J}^{-\mathbf{1}}(\ddot{\mathbf{x}}-\mathbf{J} \dot{\mathbf{q}})
\end{aligned}
$$

Combining (3), (7) and (8) $\ddot{\mathbf{q}}$ can be eliminated to give:

$$
\begin{aligned}
\mathbf{M}(\mathbf{q}) \mathbf{J}^{-1}\left(\mathbf { M } _ { \mathbf { E } } ^ { - 1 } \left(\mathbf{F}_{\mathbf{H}}-\mathbf{B}_{\mathbf{E}} \dot{\mathbf{x}}-\mathbf{K}_{\mathbf{E}}\right.\right. \\
\left.\left.\left(\mathbf{x}-\mathbf{x}_{\mathbf{E}}\right)\right)-\mathbf{J} \dot{\mathbf{q}}\right)=\boldsymbol{\tau}-\mathbf{J}^{\mathbf{T}} \mathrm{F}_{\mathrm{d}}
\end{aligned}
$$

To keep the cartesian inertia of the haptic device unchanged:

$$
\mathbf{M}_{\mathbf{E}}=\mathbf{J}^{-\mathbf{1}} \mathbf{M} \mathbf{J}^{-\mathbf{T}}
$$

Considering also slow movements typical in mouse based interaction and that $\mathbf{F}_{\mathbf{d}}=-\mathbf{F}_{\mathbf{s}}$, (9) finally gives

$$
\tau=-\mathbf{J}^{\mathbf{T}}\left(\mathbf{B}_{\mathbf{E}} \dot{\mathbf{x}}+\mathbf{K}_{\mathbf{E}}\left(\mathbf{x}-\mathbf{x}_{\mathbf{E}}\right)\right)
$$

The above equation describes the impedance control law for the haptic device. The damping and the stiffness matrixes $\mathbf{B}_{\mathbf{E}}$ and $\mathbf{K}_{\mathbf{E}}$ are $2 \times 2$ diagonal matrices and depend on the desired suppression/assistive dynamics.

Under the assistive control mode the haptic system applies assistive forces dependent on the subject's desired motion and therefore, under this mode of operation the desired direction of motion is required. 


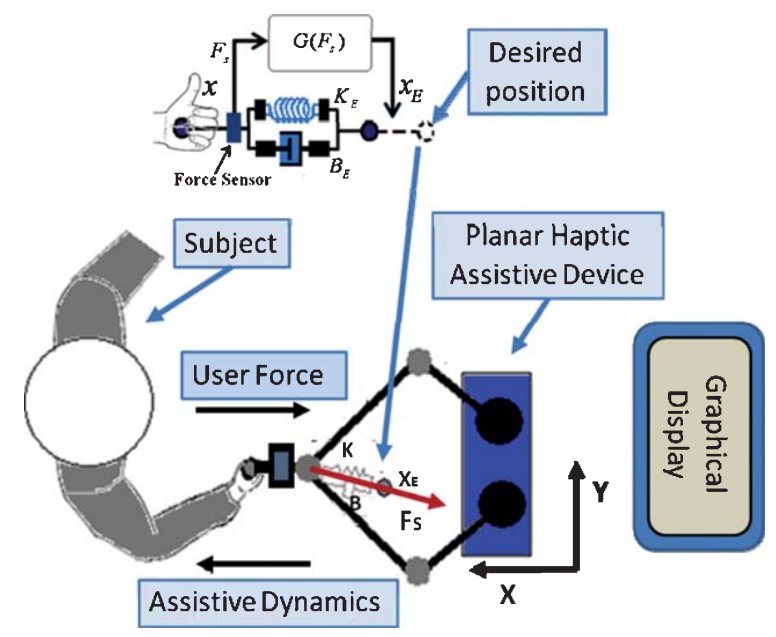

Fig. 4. System assistive mode setup.

To enable detection of the user's desired motion a Force/Torque $(\mathrm{F} / \mathrm{T})$ sensor has been mounted at the device end-tip. This sensor monitors the forces applied by the user $\mathbf{F}_{\mathbf{S}}$, Fig. 4.

Based on these force signals the desired position of the system $\mathbf{x}_{\mathbf{E}}$ is updated in (11). The following formula was used to derive the new desired position using the sensed force signal.

$$
\begin{aligned}
& x_{f}^{i}=\left\{\begin{array}{cc}
k_{a}\left(F_{S}^{i}-\alpha\right) & F_{S}^{i}>\alpha \\
0 & -\alpha<F_{S}^{i}<\alpha \\
k_{a}\left(F_{S}^{i}+\alpha\right) & F_{S}^{i}<-\alpha
\end{array}\right\} \\
& x_{E}^{i}=x_{E-1}^{i}+\int x_{f}^{i} d t, i=1 \ldots 2
\end{aligned}
$$

Where $\mathrm{F}_{\mathrm{S}}^{\mathrm{i}}$ is the ith component of the force measured by the sensor, $\mathbf{k}_{\mathbf{a}}$ is the sensitivity constant, and $\alpha$ is a noise dead band constant. By injecting the desired position vector derived from (13), into (11) assistive forces augmenting the user desired actions/motions can be generated that are governed by the stiffness matrix $\mathbf{K}_{\mathbf{E}}$ of the impedance network. At the same time the damping matrix $\mathbf{B}_{\mathbf{E}}$ of the impedance filter serves the purpose of damping erratic movements towards the direction of motion. A block diagram of the control scheme expressed by (11) and (13) is shown in Fig. 5. Where FK denotes the forward kinematics, $\mathbf{T}_{\mathbf{i}}$ refers to the motor torque constant and " $\mathbf{X}_{\mathbf{E}}$ Update Module" implements (12) and (13).

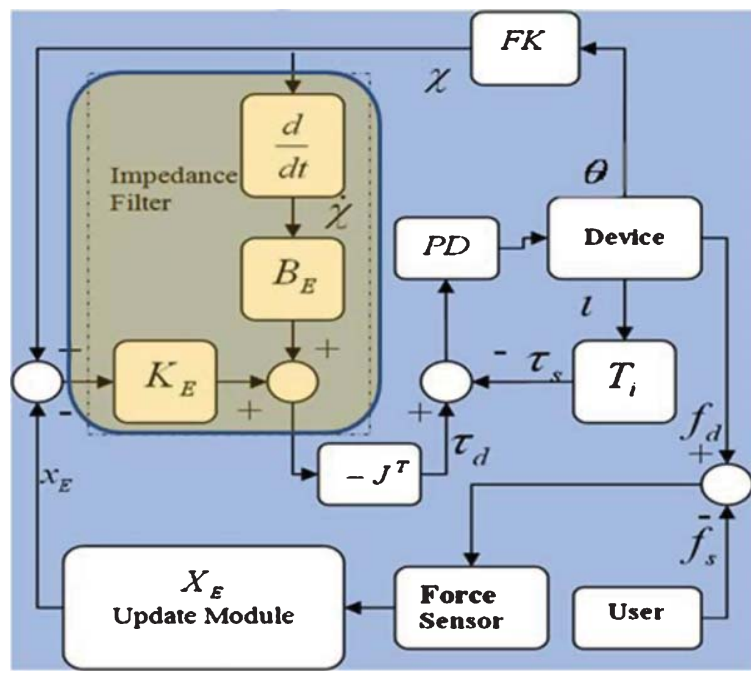

Fig. 5. Schema of the assistive control scheme.

\section{Experimental system setup and tuning}

A number of experiments were conducted to evaluate the performance of the impedance based assistive/suppression control scheme. The execution of these experiments was performed by a subject suffering from muscle ataxia. The subject, who was a daily computer user, possessed the ability to manipulate the mouse in a somewhat good manner but with difficulties in the accurate target tracking, e.g. moving a vertical or horizontal slider or selecting an item from a drop down menu. During these experiments, the subject, sitting in front of a computer display, manipulated the device tip and performed a number of target tracking tasks with and without activation of the assistive/suppression con-

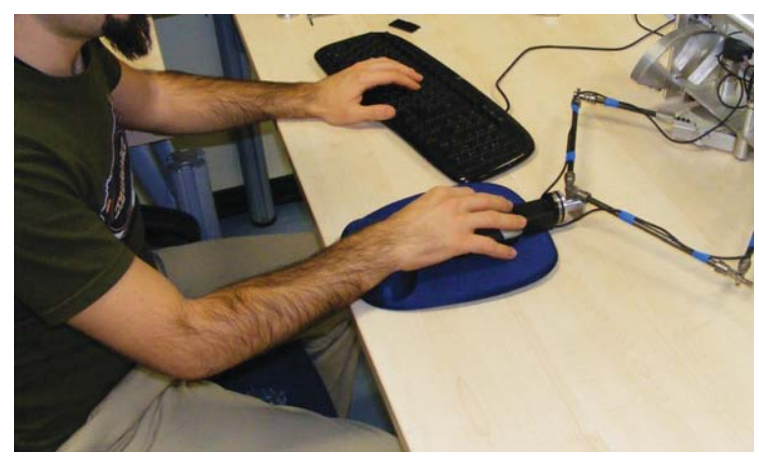

Fig. 6. Subject interacting with the mouse attached to the assistive haptic device. 
trol scheme, Fig. 6. A target tracking task required the subject to follow an on-screen target pattern with a cursor which was controlled by manipulating the tip of the haptic device in a manner similar to computer mouse input. In this experiment two tracking patterns were used, a line and a $90^{\circ}$ edge.

The execution of the tracking tasks under the assistive mode of operation required the tuning of the assistive control scheme. The inertial matrix $\mathbf{M}_{\mathbf{E}}$ of the desired impedance was set according to (10) to maintain the Cartesian inertia of the haptic system as the original. In other words, we did not modify the inertia property of the haptic system but only the damping and the stiffness properties of the impedance filter. To identify initial values for the $\mathbf{K}_{\mathbf{E}}, \mathbf{B}_{\mathbf{E}}$ matrices we performed simulation studies of the system. Through the two assistive control parameters there is independent authority over the natural frequency and damping ratio of the assistive system response (the position of the pair (Haptic device + user's hand) $\mathbf{x}$ as a function of the desired position of the system $\mathbf{x}_{\mathbf{E}}$ in (11) and (13)). From these simulations the initial guess parameters of the assistive control law $\mathbf{K}_{\mathbf{E}}, \mathbf{B}_{\mathbf{E}}$ were selected considering the desired bandwidth and damping ratio of the assistive system. Since the presented system and control are utilized as a mouse based motion assistive device it was coherent to select the $\mathbf{K}_{\mathbf{E}}, \mathbf{B}_{\mathbf{E}}$ such that the closed loop bandwidth is greater than the maximum motion frequency during mouse based interaction movements $(2-3 \mathrm{~Hz}$ or $12-18 \mathrm{rad} / \mathrm{sec})$. In these trials we consider a bandwidth of $5 \mathrm{~Hz},\left(\omega \_\mathrm{n} \sim 30 \mathrm{rad} / \mathrm{sec}\right)$ and a damping ratio of $s=\sqrt{2} / 2$ for the assistive response of the $2 \mathrm{DOF}$ system presented in Fig. 4. The initial guess assistive network matrices were computed as $\mathbf{K}_{\mathbf{E}}=\left[\begin{array}{ll}\mathrm{k} & 0 \\ 0 & \mathrm{k}\end{array}\right]$, $\mathrm{k}=3.7 \mathrm{~N} / \mathrm{m}$ and $\mathbf{B}_{\mathbf{E}}=\left[\begin{array}{ll}\mathrm{b} & 0 \\ 0 & \mathrm{~b}\end{array}\right], \mathrm{b}=0.3 \mathrm{Nsec} / \mathrm{m}$. Further tuning of the above stiffness and damping matrixes was performed by a series of trials where the impaired subject manipulating the device was asked to move randomly in the XY plane while adjustments of the $\mathbf{K}_{\mathbf{E}}, \mathbf{B}_{\mathbf{E}}$ were applied. By using the force level data applied to the load cell and the feedback from the subject the scalar values of the diagonal stiffness and damping matrixes $\mathbf{K}_{\mathbf{E}}$ and $\mathbf{B}_{\mathbf{E}}$ were finally adjusted to $\mathrm{k}=2.5 \mathrm{~N} / \mathrm{cm}, \mathrm{b}=0.5 \mathrm{Nsec} / \mathrm{cm}$ respectively compromising between user comfort, erratic motion suppression and assistance. Finally a dead band force threshold of $0.4 \mathrm{~N}$ was applied in (12) to prevent any force sensor noise from affecting the update of the desired position of the system $\mathbf{x}_{\mathbf{E}}$ in (11).

\section{Results and discussion}

The tracking difficulty of the muscle ataxia subject is demonstrated in Fig. 7 which shows the rough trajectories generated while performing two dimensional target tracking tasks. During this experiment no haptic assistance was provided.

The impaired subject was asked to track the line and edge patterns several times as fast as they could. The average execution time for one pass and the average standard deviation of the trajectory from the reference pattern were used to evaluate the tracking performance. Observing the impaired subject trajectory in Fig. 7, it
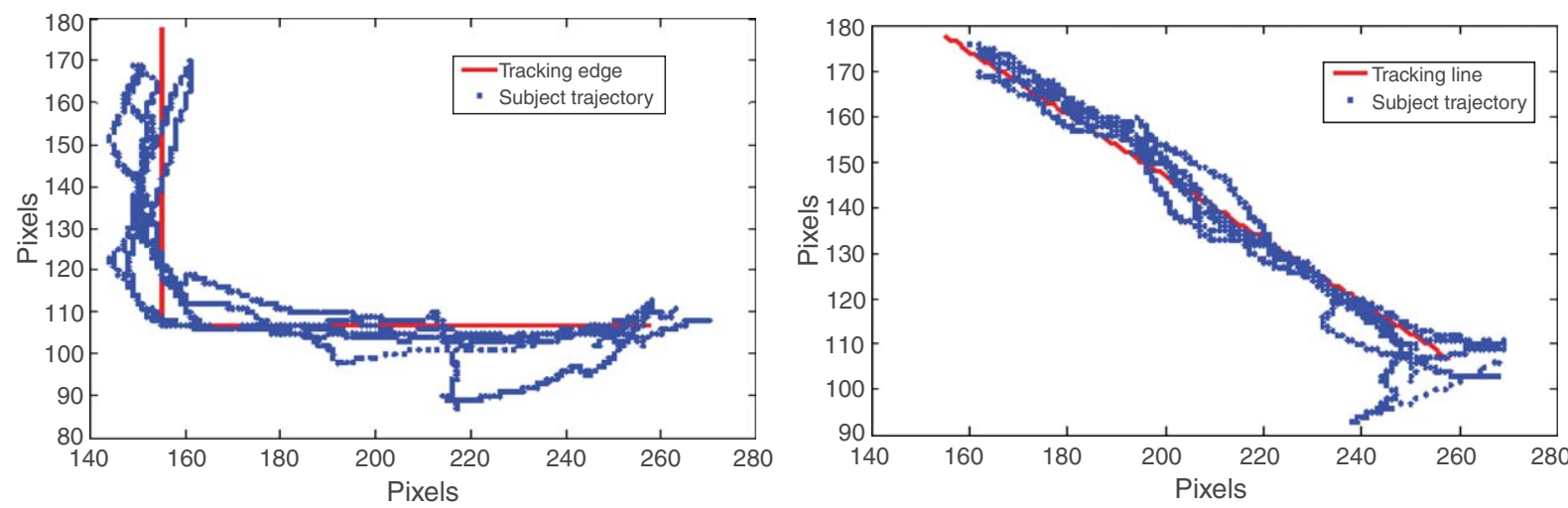

Fig. 7. Line and edge tracking performance of the muscle ataxia impaired subject. 
is evident the existence of erratic motions which result to very different trajectories among the several trials. For the corner profile these erratic motions occur at all stages of the motion while for the diagonal line profile the effect was particularly observed at the endpoints. To demonstrate the erratic and random tracking performance and the variation among the several trajectories we compute the distance error between the points of the reference pattern and the points of each trial trajectory. We perform a one-way ANOVA to compare them with the null hypothesis being that the error distances do not differ significantly on average among the several trials. In the case of edge pattern the test return $\left(p<0.05, F_{(4,169)}=31.9\right)$ showing a strong evidence that the error distances and the trends of the trajectories during the several trials differ significantly. Similarly for the case of the line pattern the one-way ANOVA test return $\left(p<0.05, F_{(4,104)}=155.5\right)$ concluding in the same result. The high variation among the several trials is a direct consequence of the unsteady and randomly occuring clumsy motions resulted from the muscle ataxia impairment.

Following this the haptic display mode was set to assistive mode using the control strategy presented in section III and the same experiment was executed. Fig. 8, show the tracking trajectory for the line and the edge tracking tasks. It can be seen in Fig. 8 that the amplitude of the sudden motions has been reduced with the application of the assistive control. Comparing the performance measures of these trajectories with those obtained without the assistance it can be seen that in the case of the edge pattern the relevant measures of the assisted trajectory $(\sigma=10.64, t=14.72)$ were superior to those of the unsupported trajectory $(\sigma=18.91, t=18.13 \mathrm{~s})$. They are evident the improved smoothness and the reduction of the amplitude of the erratic motions which finally result in better tracking performance.

In the case of the line target although the standard deviation of the trajectory $(\sigma=17.9)$ is very similar to the one achieved without the assistance in Fig. 7, $(\sigma=18.25)$, the overall trajectory is also more smooth and regular with fewer and smaller jerks or spasms. In terms of execution time the subject was able to complete the task in less time $(t=8.71 \mathrm{~s})$ compared to $(t=11.89 \mathrm{~s})$.

In addition, we perform a $t$-test between the means of the error distances of the tracking trajectories without and with haptic assistance. Both for the case of the edge and the line patterns the $t$-test indicated significant differences on the mean distance errors $(p=0.026$, $t_{(4)}=3.43$ for the edge $)$ and $\left(p=0.011, t_{(4)}=4.45\right)$ for the line pattern. The mean error distance of the trajectories with haptic assistance (w) for both the edge and the line pattern were significant lower compared to those without (wo) the haptic assistance $(\mathrm{w} / \mathrm{wo}=11.64 / 16.31$ for the edge $)$ and $(\mathrm{w} / \mathrm{wo}=$ $6.09 / 16.08$ for the line pattern).

These preliminary results indicate that some performance improvements in terms of time required to perform a task and accuracy in performing the tracking task can be gained when the assistive/suppression impedance control is active. However, more extensive evaluation is necessary to state this categorically. The qualitatively assessed overall impression for the functionality of the system as reported by the subject was in general positive. Some comments related to the different hand grasping posture when compared
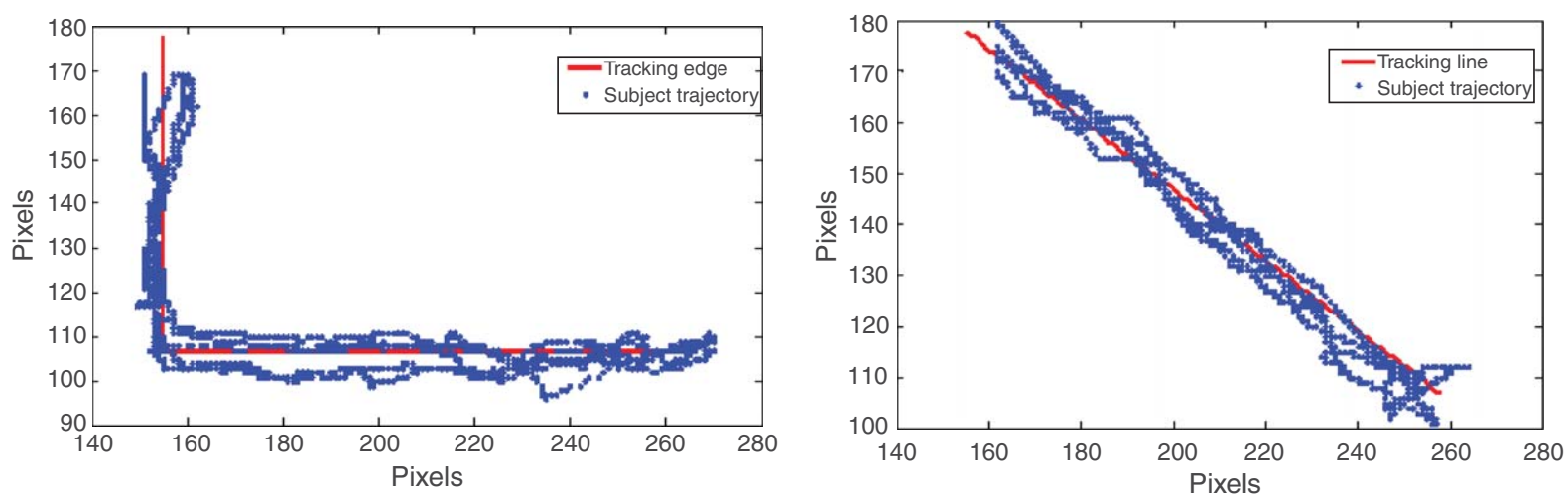

Fig. 8. Line and edge tracking performance of the impaired subject with assistive control. 
to traditional mouse grasping posture were mentioned. This is considered as no significant issue considering the early development stage of the system and will be addressed in the future work.

\section{Conclusions}

In this work a system is proposed for assisting impaired subjects having coordination difficulties in performing two dimensional tracking tasks. The system employs a $2 \mathrm{DOF}$ pantograph planar device that allows the interaction of the user with a computer generated environment in a manner analogous to computer mouse interactions. The control scheme of the device was designed to help an impaired subject with motion coordination difficulties to complete a tracking task with higher accuracy and in less time. In particular the control scheme serves two main purposes, assisting the motion in the case of a person with weakened muscles, and damping unsteady motion intervals in the direction of the movement. To achieve the above purposes an impedance assistive/suppression control scheme was used. The overall system was evaluated with experimental trials performed by an impaired subject suffering from muscle ataxia. In these experiments the subject performed tracking tasks involving two primitive tracking patterns, a line and a $90^{\circ}$ corner. Despite the fact that the presented system has been evaluated with only a single the preliminary results obtained from these experiments are still promising and valuable as demonstrated clear improvement in the subject performance. The tracking performance with assistance improved in terms of both the execution time and the standard deviation of the trajectory from the reference tracking target. This significant improvement observed with the single subject participated is the motivation for further studies as required to state the benefits of the system categorically. These studies will include the following:

- Extensive assessment of the system with more subjects.

- Further development of the control architecture with the incorporation of an adaptation agent to allow self tuning of the system according to the user needs.

- Interfacing of the haptic device tip with a computer mouse to allow natural interaction with GUI controls.

\section{References}

[1] S. Trewin and H. Pain, Keyboard and mouse errors due to motor disabilities, International Journal of Human-Computer Studies 50(2) (1999), 109-144.

[2] J.T. Dennerlein and M.C. Yang, Haptic force feedback devices for the office computer: Performance and musculoskeletal loading issues, Human Factors 43(2) (2001), 278-286.

[3] S. Hasser, A. Goldenberg, K. Martin and L. Rosenberg, User performance in a GUI pointing task with a low-cost force feedback computer mouse, in: Proceedings of the ASME Dynamic Systems and Control Division, American Society of Mechanical Engineers, 1998, pp. 151-156.

[4] F. Hwang, S. Keates, P. Langdon and P.J. Clarkson, Multiple haptic targets for motion impaired users, in: Proceedings of CHI 2003, Fort Lauderdale, FL, 2003, pp. 41-48.

[5] S. Beringhause, M. Rosen and S. Haung, Evaluation of a damped joystick for people disabled by intention tremor, in: Proc RESNA 12th Annu Conf' 89 , pp. 41-42.

[6] M. Rosen, A. Arnold, I. Baiges, M. Aisen and S. Eglowstein, Design of a controlled-energy-dissipation-orthosis (CEDO) for functional suppression of intention tremors, J Rehab Res Dev 32(1) (1995), 1-16.

[7] B. Morrice, W. Becker, J. Hoffer and R. Lee, Manual tracking performance in patients with cerebellar incoordination Effects of mechanical loading, Canadian J Neurol Sci 17(3) (1990), 275-285.

[8] B. Cesqui, S. Micera, S. Mazzoleni, M.C. Carrozza and P. Dario, Analysis of upper limb performance of elderly people using a mechatronic system, Proceedings of BioRob 2006, pp. 365-370.

[9] M. Uemura, K. Kanaoka and S. Kawamura, Power assist system for SinusoidalMotion by passive element and impedance control, Proceeedings of IEEE Robtics and Automation, Orlando, Florida, May 2006, pp. 3935-3940.

[10] T. Nef, M. Mihelj, G. Colombo and R. Riener, ARMin Robot for rehabilitation of the upper extremities, Proceedings of the 2006 IEEE International Conference on Robotics and Automation, Orlando, Florida, May 2006, pp. 3152-3157.

[11] E. Rocon, A.F. Ruiz, F. Brunneti and J.L.Pons, On the use of an active wearable exoskeleton for tremor suppression via biomechanical loading, Proceedings of the 2006 IEEE International Conference on Robotics and Automation, Orlando, Florida, May 2006, pp. 3140-3145.

[12] K. Kiguchi, T. Tanaka, K. Watanabe and Fukuda, Design and control of an exoskeleton system for human upper-limb motion assist, Proceedings of AIM, 2003, pp. 926-931.

[13] K. Kong and D. Jeon, Design and control of an exoskeleton for the elderly and patients, Mechatronics 11(4) (Aug. 2006), pp. $428-432$.

[14] M.B. Delatycki, R. Williamson and S.M. Forrest, Friedreich ataxia: An overview, J Med Genet 37 (2000), 1-8.

[15] N.G. Tsagarakis, M. Petrone, D. Testi, R. Mayoral, C. Zannoni, M. Viceconti and G.J. Clapworthy D.G. Caldwell, Pre-operative planning for total hip arthroplasty using a haptic enabled multimodal interface and framework, IEEE Multimedia and Visualization: Special Issue in Haptics, July-Sept. 2006, pp. 40-48.

[16] N.G. Tsagarakis and D.G. Caldwell, A 5 dof Haptic Interface for Pre-Operative Planning of Surgical Access in Hip Arthroplasty, WordHaptics, Pisa, Italy, 2005, pp. 519-524. 

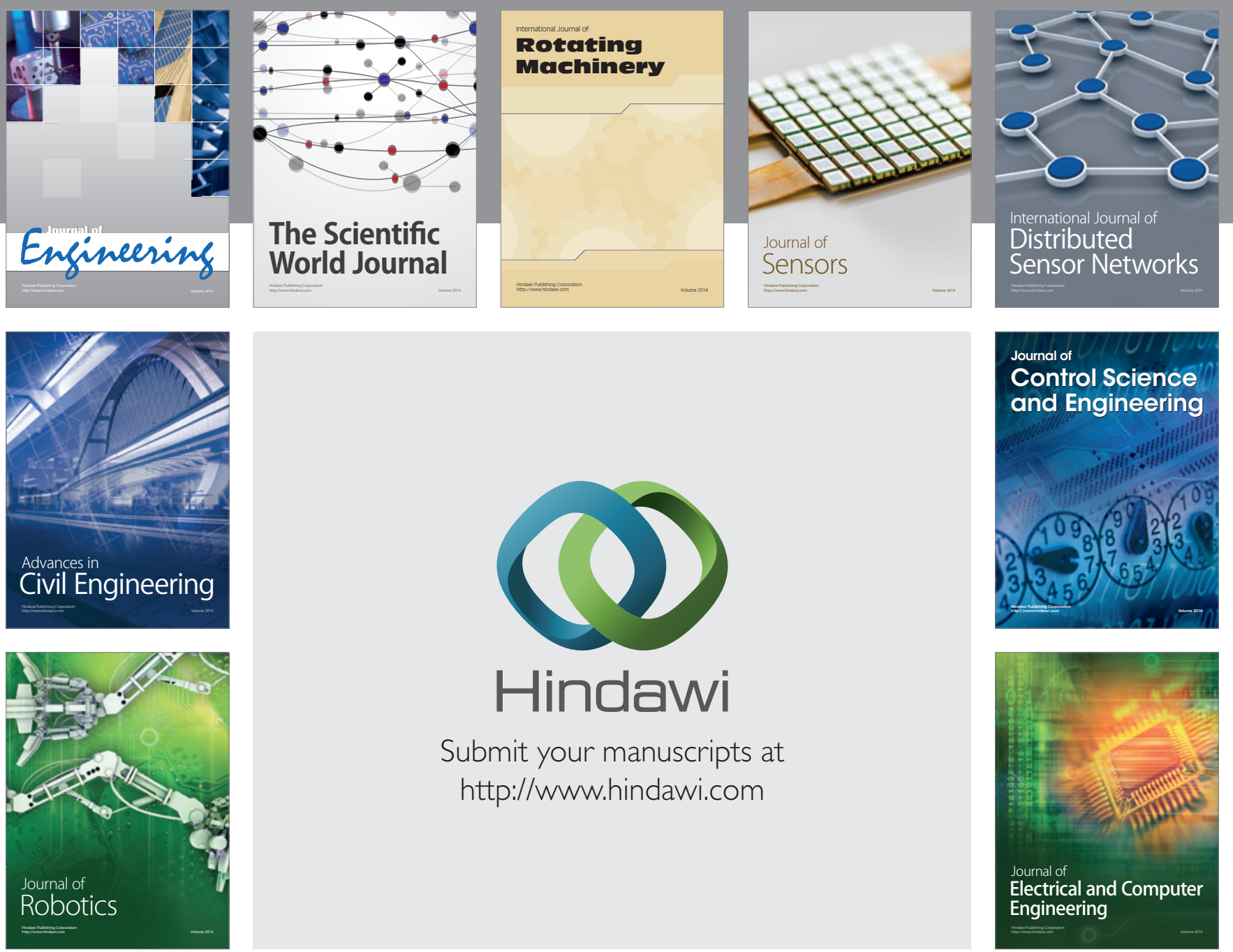

Submit your manuscripts at

http://www.hindawi.com
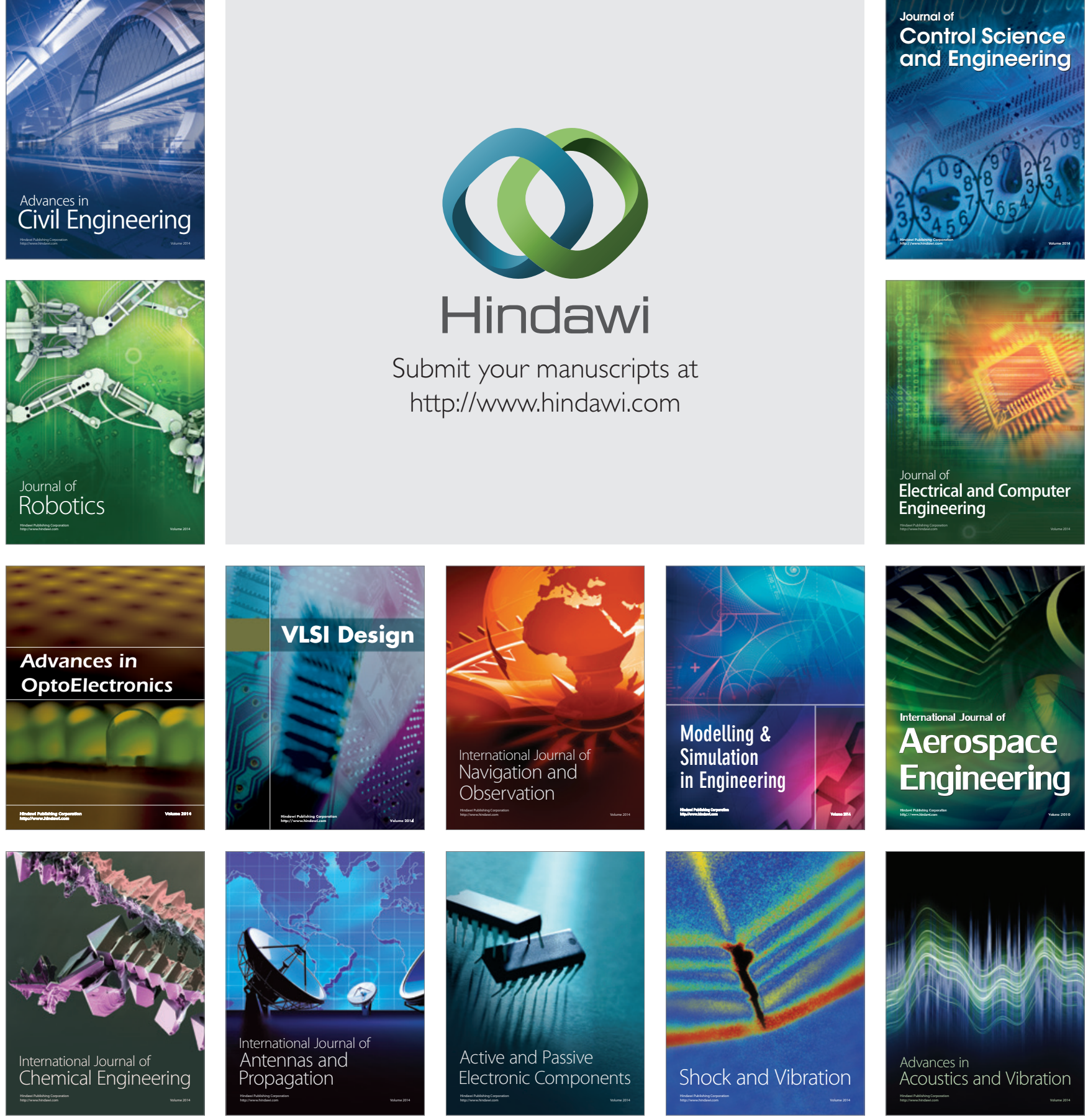